\title{
评述
}

\section{基于质子MRSI的肌细胞内脂测量技术的原理、 现状及展望}

李敏 ${ }^{1,2 *}$, 张学武 ${ }^{1,2}$, 沈文荣 ${ }^{3}$, 邓志祥 ${ }^{1}$, 董正超 ${ }^{2,4^{*}}$

1. 河海大学物联网工程学院, 常州 213022;

2. Division of Molecular Imaging, Department of Psychiatry, Columbia University, New York 10032, USA;

3. 江苏省肿瘤医院影像中心, 南京 210009 ;

4. New York State Psychiatric Institute, New York 10032, USA

*联系人, E-mail: 1m_0711@163.com; zd2109@cumc.columbia.edu

收稿日期：2018-11-30; 接受日期：2019-06-27; 网络版发表日期：2019-11-08

国家自然科学基金(批准号: 61671202, 61573128, 61701169)和江苏省重点研发项目(批准号: BE2017071, BE2017647)资助

摘要胰岛素抵抗作为诱发糖尿病的主要原因可能早在糖尿病发病十多年前就已经出现. 及早发现胰岛素抵抗 对于糖尿病的早期预防、诊断、治疗有着至关重要的意义. 研究发现, 人类小腿肌肉细胞内脂质(intramyocellular lipid, IMCL)的含量与胇岛素抵抗和代谢障碍有着密切而又复杂的关联. 因此, 准确测量IMCL对于 II 型糖尿病乃 至多种精神疾病具有重要的研究意义。然而，目前测量IMCL的“金标准”是创伤性的活检技术，不适于大规模的基 础研究和长期的临床应用. 基于质子磁共振波谱成像 $\left({ }^{1} \mathrm{H}\right.$ magnetic resonance spectrosocpic imaging, $\left.{ }^{1} \mathrm{H} \mathrm{MRSI}\right)$ 的 IMCL测量技术提供了一种无辐射、无创伤、高精度的检测方法, 有望成为明晰IMCL含量与胰岛素抵抗, 以及胰 岛素抵抗与 II 型糖尿病关系的重要技术并在临床上应用。本文基于 ${ }^{1} \mathrm{H}$ MRSI的IMCL含量测量技术，阐述并总结 了其发展历程，从小腿内部生理结构特征、肌纤维方向、脂肪分布、精确量化IMCL含量等方面指出目前该技术 面临的挑战和最新的解决思路. 本文特别探讨了多模态磁共振成像(magnetic resonance imaging, MRI)技术以及面 向参数估计的磁共振指纹技术MRF(magnetic resonance fingerprinting)等新技术与质子磁共振波谱成像技术的结 合, 希望为 IMCL高精度测量提供更加多样化、多维度、多尺度的可能和途径.

关键词磁共振波谱成像, IMCL, EMCL, 糖尿病, 肌肉纤维方向, 多模态磁共振成像, 磁共振波谱指纹技术

糖尿病是一种严重危害人类身体健康和生活质量 的非传染性疾病，在全世界不断曼延。糖尿病不可治 愈，且容易导致许多并发症(如心脏病、肾衰竭以及失 明等), 会给个人、家庭及社会带来巨大的精神损失和 经济负担 ${ }^{[1 \sim 3]}$. 世界卫生组织指出 ${ }^{[4]}$ : 全球糖尿病人数
量已从1980年的 1.08 亿增长到 2017 年的 4.3 亿; 未来 20 年糖尿病还将迅速曼延, 而发展中国家将成为“重灾 区”。如今，我国的糖尿病患者人数居全球第一，截至 2017 年已达到1.144亿, 且呈低龄化、快速化的发展 趋势 ${ }^{[5]}$.

引用格式: 李敏, 张学武, 沈文荣, 等. 基于质子MRSI的肌细胞内脂测量技术的原理、现状及展望. 中国科学: 生命科学, 2020, 50: 14-24 Li M, Zhang X W, Shen W R, et al. The principle, present and prospect of IMCL measurement based on ${ }^{1} \mathrm{H}$ MRSI technology (in Chinese). Sci Sin Vitae, 2020, 50: 14-24, doi: 10.1360/N052018-00278 
虽然目前糖尿病本身不可治愈，但及早发现(至少 可以提前 10 年)、干预和治疗，可以有效避免许多并发 症(心脏病、肾衰竭以及失明等)的发生 ${ }^{[6,7]}$. 然而, 我国 在糖尿病的发现、诊断和治疗能力上还很薄弱. 糖尿 病患者中，诊断率不足 $47 \%$ ，治疗率仅为 $25.8 \%$. 如何 有效控制糖尿病的发展, 提高糖尿病的治疗水平, 成为 我国医疗卫生系统面临的严峻挑战.

研究发现, 胰岛素抵抗(出现在发病10 20年前, 且 与胰岛素含量无关)即细胞对胰岛素不敏感是糖尿病 发病的主要原因 ${ }^{[8 \sim 10]}$. 研究报道显示, 人类小腿肌肉的 细胞内脂质(intra-myocellular lipids, IMCL)水平与胰 岛素抵抗、II 型糖尿病有密切而复杂的关系 ${ }^{[114]}$. 准 确测量IMCL水平的变化，对胰岛素抵抗的早期发现， 糖尿病的预防、管理，改善糖尿病患者的生活质量， 延缓并发症的产生等都具有重要的意义 ${ }^{[15,16]}$.

传统活检技术(利用提取活体组织测量IMCL含 量), 因其有创性、精确度低等问题，不适于大规模、 长时间的实验研究和同一病人的多部位、重复性、长 期跟踪性的临床应用 ${ }^{[17,18]}$. 为此, 利用质子磁共振波谱 成像 $\left({ }^{1} \mathrm{H}\right.$ magnetic resonance spectral imaging, ${ }^{1} \mathrm{H}$ MRSI $)$ 技术测量人体小腿肌细胞内脂质含量的方法，以其无 创伤、非辐射、可多部位、重复观测、操作复杂度 低、易于推广等优势, 受到学术界的广泛关注 ${ }^{[19]}$.

然而，利用质子磁共振波谱成像技术测量人体小 腿肌细胞内脂质含量的精度受到多种因素的影响. 主 磁场的不均匀性、体磁化率效应、细胞外脂质(extramyocellular lipids, EMCL)干扰所引起的MRS谱线平 移、加宽和畸变, 都会使IMCL谱线与EMCL谱线重 叠, 甚至淹没在EMCL畸变谱线中, 难以有效区分, 影 响测量结果的准确性. 这种情况对潜在的糖尿病患者 更为突出 ${ }^{[20]}$. 另外, 如何精确测量IMCL以及其他相关 代谢物质的方法还有待进一步的研究.

近年来，磁共振成像(magnetic resonance imaging, MRI)技术不断丰富与发展. 联合运用核磁共振波谱成 像技术与多模态MRI技术(包括结构MRI、脂肪MRI、 弥散张量成像、磁共振指纹技术即MRF(magnetic resonance fingerprinting)), 将有助于增加量测空间信息 维数, 提高量测对象特征描述的准确性及测量的可信 度, 为提高IMCL的测量精度带来新的解决思路 ${ }^{[21 ~ 23]}$.

本文首先介绍了传统的IMCL测量技术及其局限 性, 引出基于 ${ }^{1} \mathrm{H}$ MRSI的肌肉细胞内脂质测量技术的基
本原理; 其次就 ${ }^{1} \mathrm{H}$ MRSI测量肌肉细胞内脂质含量技术 需要克服的难题及其解决的方法进行了回顾和评述; 然后针对目前研究过程中该技术面临的新挑战以及新 机遇进行深入分析和探讨; 最后总结并展望了未来细 胞内脂质IMCL精准测量技术在糖尿病应用上的前景.

\section{IMCL检测技术概况}

每年我国有近 100 万的糖尿病患者死亡，其中 $40 \%$ 患者不满70周岁. 随着人民生活水平的不断提升和生 活方式的改变, 食用过于精细的食物且缺乏足够的运 动, 使得糖尿病的发病率持续增长. 而缺乏有效、便 捷的检测手段成为目前糖尿病蔓延、难以控制的主要 原因 ${ }^{[24]}$.

\section{1 传统的生化检测方法}

传统IMCL的测量方法是肌肉活检, 即从身体提取 活组织(约 $0.1 \mathrm{~g}$ )进行病理学测量. 肌肉活检技术的具 体方法: 活检方法通常选择较易分离细胞的活体组织 作为样本 (例如，人体的小腿部位肌肉和脂肪分布清 晰, 比较适合该项检测), 然后由人工将细胞的外脂质 剥离, 使用电子显微镜观察超薄切片 $(10 \mathrm{~nm})$ 中肌肉细 胞内脂滴含量. 通过随机抽取若干视野进行脂滴数量 计数, 把平均单视野内脂滴数量的平均数作为肌肉细 胞内脂肪含量, 对细胞内脂质含量进行测量.

活检后最常见的问题是疼痛. 英国伦敦大学学院 医院指出, 疼痛通常持续几天到几周. 与此同时, 活检 部位的疼伤和不适，出血时间延长以及感染等都是该 技术存在的主要风险 ${ }^{[17,18]}$. 为此，活检方法通常不能 多点、多次测量, 不适应于实时IMCL动态观测, 而这 点对于评价治疗效果、调整治疗方案具有重要的参考 意义. 另外, 由于肌细胞内脂质含量远远小于肌细胞外 脂质的含量, 活检方法很难精确地分离细胞内脂质 $\mathrm{IMCL}$ 与细胞外脂质 $\mathrm{EMCL}$, 导致测量误差增大(准确 度仅能达到 $20 \% \sim 30 \%)^{[20]}$.

这些方法除了具有侵入性以外，还存在技术复 杂、过程冗长、价格昂贵的问题，因而不适于临床检 验和大型实验研究 ${ }^{[25]}$.

\section{2 基于 ${ }^{1} \mathrm{H}$ MRSI的IMCL测量技术}

(1) IMCL与EMCL谱线的分离. 利用质子磁共振 
波谱 ${ }^{1} \mathrm{H}$ MRSI测量IMCL含量的基本原理在于IMCL与 EMCL在谱峰上的可分离性. IMCL和EMCL有着相同 的化学成分一一脂肪酸或甘油三酯. 但是，两者在肌 肉中的微结构形态和位置均存在一定的差异. IMCL 是以脂肪微滴(lipid droplets)的形式存在于肌细胞内, 而EMCL则是以片状或管状的脂肪渗透存在于肌肉纤 维间或筋膜中. 用装在毛细管中的豆油来模拟EMCL, 同时用球形容器中的脂肪乳模拟 IMCL, 分别进行 ${ }^{1} \mathrm{H}$ MRSI测量, 可以证实, EMCL的磁共振谱线的位置 随毛细管(模拟肌纤维)取向与主磁场方向夹角 $\theta$ 而移 动，而IMCL的谱线位置则不改变. 这就是磁共振理论 中的体磁化率效应(bulk magnetic susceptibility, $\mathrm{BMS})^{[26]}$. EMCL谱线位移与夹角 $\theta$ 之间的对应变化关 系可表示为

$\mathrm{d} f_{\mathrm{EMCL}}=1.2 \times\left(3 \times \cos ^{2} \theta-1\right)(\mathrm{ppm})$.

图1为计算机模拟的肌肉纤维方向与主磁场方向 成不同角度 $\theta$ 时, EMCL和IMCL磁共振波谱曲线的相 对位置. 模拟所用的参数: EMCL与IMCL的含量比为 $2: 1$, 线形为洛伦兹, 线宽为 $10 \mathrm{~Hz}$, 场强为 $3 \mathrm{~T}$. 如图所 示, 当 $\theta=0^{\circ}$ 时, $\mathrm{EMCL}$ 与 IMCL的谱线距离最大, 约为 $0.24 \mathrm{ppm}$; 当 $\theta=54.7^{\circ}$ 时，两者完全重合; 当 $\theta$ 角散开时, EMCL的谱线会因频移而加宽. 由此可知，当 $\theta$ 角较小 时，EMCL的BMS效应使得利用质子磁共振波谱分离 $\mathrm{EMCL} / \mathrm{IMCL}$ 成为可能. 但与此同时, 肌肉纤维方向的 分散，即 $\theta$ 角的散开也会加大EMCL的线宽，从而为 $\mathrm{EMCL} / \mathrm{IMCL}$ 谱线的分辨带来困难.

（2）IMCL浓度的相对及绝对定量测量. 利用 ${ }^{1} \mathrm{H}$ MRS测量IMCL含量, 在准确地将EMCL和IMCL谱 线分离后, 还需要将IMCL谱线对应的峰面积转换成相 应单位下的浓度值. 目前大多数方法是选择合适的参 照物，根据 ${ }^{1} \mathrm{H}$ MRS测量到的信号，将IMCL的浓度表 达为IMCL相对于参照物信号的相对值, 这称为相对定 量测量; 也可以在精确获知参照谱线所对应单位体积 浓度的前提下, 对 ${ }^{1} \mathrm{H}$ MR 测得的IMCL信息与参考物信 号进行必要的校正, 推算出IMCL谱线面积对应物质的 绝对含量, 这称为绝对定量测量.

常用参照物可以是内部的, 也可以是外部的. 常用 的内部参照物可以采用肌肉、皮下脂肪、骨髓和肌酸 等物质中的局部水分子信号 ${ }^{[27 ~ 29]}$. 这类参照物的信号

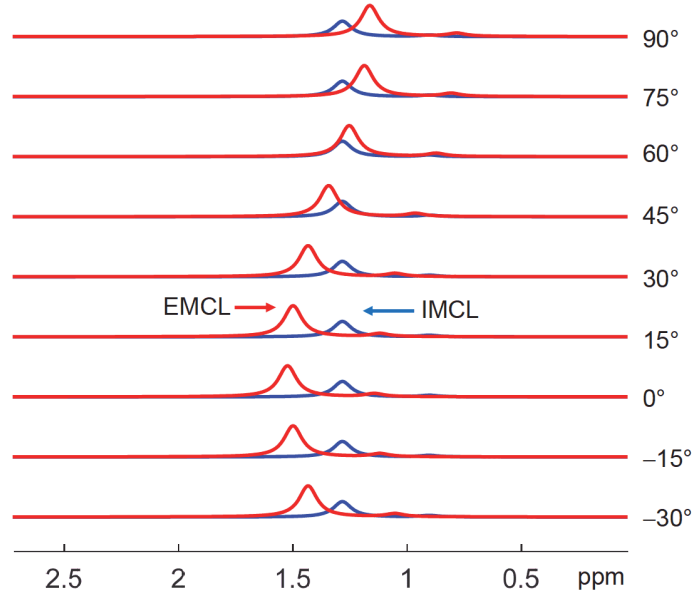

图 1 BMS效应模拟实验的示意图

Figure 1 Simulation experiment of BMS

是与IMCL信号在相同的实验条件下获得的. 外部参 照物则是利用装在小瓶里的水油混合物的信号. 此类 参照物可以准确测量水或脂肪的浓度，但实验条件的 差异性可能给IMCL的定量标定带来附加的误差. 下 面以内部参照物(internal reference)为例分别说明相对 和绝对定量测量的方法.

设 ${ }^{1} \mathrm{H}$ MRS波谱信号 $S$ 由 $M$ 个分子，包括水、脂 肪、代谢物的信号组成，其中任一分子 $m$ 的信号大小 (或谱峰的面积)可表示为 ${ }^{[30]}$

$$
\begin{aligned}
S_{m} & =k C_{m} V\left(1-\mathrm{e}^{-T R / T_{1, m}}\right) \mathrm{e}^{-T E / T_{2, m}} \\
& =S_{0, m}\left(1-\mathrm{e}^{-T R / T_{1, m}}\right) \mathrm{e}^{-T E / T_{2, m}},
\end{aligned}
$$

其中, $T_{1}$ 和 $T_{2}$ 分别是纵向和横向弛豫时间, $T E$ 和 $T R$ 分 别是回波和重复时间, $C$ 是浓度, $V$ 是MRS体素的大小 (假设它对所有分子是相同的), $k$ 是其他由实验条件(包 括磁场强度、接收线圈增益等)所决定的常数.

若以水为参照物，则某一待测物的相对浓度可表 示为

$\left[C_{m}^{*}\right]=\frac{S_{m}}{S_{w}}\left[C_{w}^{*}\right]$.

如果给出 $C_{w}^{*}$ 的值, 则 $C_{m}^{*}$ 得出相应的浓度值和单 位, 否则, $C_{m}^{*}$ 为机构单位(institutional unit)的浓度值. 而绝对浓度可由下式求出:

$\left[C_{m}\right]=\frac{S_{0, m}}{S_{0, w}}\left[C_{w}\right]$, 
其中 $S_{0, m}, S_{0, w}$ 已知其弛豫时间的情况下对 $S_{m}$ 和 $S_{w}$ 根据 (2)式进行校正得出. 如果使用外部参考物(external reference), 则需要对 $S_{w}$ 中影响 $k$ 的所有实验参数进行校 正. 这增加了难度和误差 ${ }^{[19,31]}$.

\section{2 基于 ${ }^{1} \mathrm{H}$ MRSI的IMCL 测量方法研究 进展}

基于 ${ }^{1} \mathrm{H}$ MRSI的IMCL测量方法, 可以满足糖尿病 早期预测、诊断和治疗过程中多次重复性观测的需 求, 大大降低测量成本和病人痛苦, 是一种便捷、可靠 的测量方法 ${ }^{[32]}$. 然而, 主磁场不均匀性、肌纤维细胞 方向变化多样性以及皮下脂肪强信号干扰等因素，均 为EMCL与IMCL的MRS谱线分离以及IMCL含量的准 确测量造成困难. 本文从三个主要方面阐述这些困难 和克服这些困难的技术研究进展.

\section{1 肌纤维方向的离散性}

根据体磁化效应, 当肌纤维平行于主磁场时, 细胞 内、外脂质(IMCL-EMCL)的磁共振谱线裂分最大, 最 容易区分. 然而, 在较大体积的肌肉块中, 肌纤维的方 向不可能完全一致，而是具有一定的分布特性，如图2 所示.

理想情况下, 肌纤维方向一致, 它与主磁场B方向 之间的夹角 $\theta$ 直接决定了EMCL谱线的偏移量，但并不 造成谱线的加宽和线形畸变; 然而实际情况中, 肌纤维 的分布方向并非一致，如图2右侧图所示. 肌纤维方向 与主磁场之间夹角的分散，会造成EMCL谱线加宽和 线形畸变, 即IMCL谱线与 EMCL谱线的间距小于 $0.24 \mathrm{ppm}$ 并使EMCL谱线偏离可解析的理论线形. 这 为IMCL与EMCL的分辨带来困难 ${ }^{[26,33]}$.

若能对EMCL的畸变谱线进行拟合, 即可以实现 IMCL含量的测量. 但由于EMCL谱线畸变的非对称 性, 经典的对称型解析函数线形(如高斯线形和洛伦兹 线形)无法对其进行准确的拟合. 为此, 研究者从模拟 肌群纤维分布的角度估计EMCL谱线的畸变, 重建 EMCL的线形 ${ }^{[21]}$. 该方法首先对所考虑的体元(voxel) 中的肌群纤维角度分布做如下的假设: 肌群纤维的方 向相对于主磁场成高斯分布(参照图2), 可以用高斯分 布函数表示:

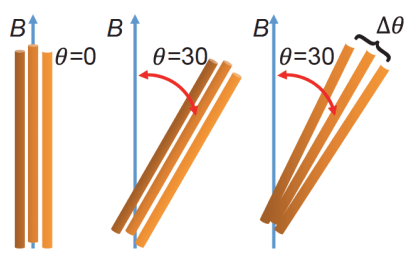

图 2 肌纤维方向的离散性示意图

Figure 2 A schematic diagram of the orientations of muscle fibers

$N(\theta, \sigma)=\mathrm{e}^{-\left(\frac{\theta-\theta_{c}}{\sigma}\right)^{2}}$,

其中参数 $\theta_{c}$ 为肌肉纤维最可取的方向 $0<\theta_{c}<\pi / 2, \sigma$ 为 纤维方向的分布宽度. 在 $\theta_{c}$ 和 $\sigma$ 确定的情况下, $\theta$ 附近的 纤维“条数”为

$\mathrm{d} N(\theta, \sigma)=\mathrm{e}^{-\left(\frac{\theta-\theta_{c}}{\sigma}\right)^{2}} \mathrm{~d} \theta$,

其中谱线的位置由式(1)决定，谱线的强度正比于 $\mathrm{d} N$. 由式(6)求定积分就可得到这个肌肉纤维分布的EMCL 线形. 这个线形可用于谱线拟合. 但是, 线形的参数 $\left(\theta_{c}, \sigma\right)$ 是未知的, 需要在谱线拟合时与其他谱线参数 一起不断地改变来实现最优拟合, 从而确定EMCL和 IMCL的谱线参数, 计算出IMCL谱线的面积. 这一方 法可以提高谱线拟合的精度, 但由于增加了未知参数, 拟合的稳健性会受到影响.

受这一方法的启发, 研究者不断提出, 借鉴多模态 MRI技术来解决IMCL测量问题. 例如, 借助弥散张量 成像(diffusion tensor imaging, DTI), 获得纤维走向的

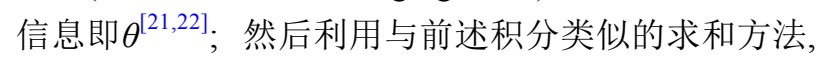
重建EMCL谱线线形. 图3为该方法主要思想示意图. 假设MRS体元内包括了 $4 \times 4$ 个DTI体元 (实际上MRS 体元的体积为立方厘米量级, 而DTI体元的大小为立 方毫米量级, 因此每个MRS体元包括的DTI体元个数 约为 $10^{3}$ 个), 其中每个张量椭球给出了肌肉纤维与磁 场的夹角. 根据这个夹角和式(1)可算出EMCL的线移. 将这些小谱线加起来就可得到MRS体元的线形. 这个 线形可直接用于谱线拟合. 这一方法需要从实验上测 量 $\theta$ 的分布, 稳健性好, 但是DTI扫描增加了约 10 分钟 的测量时间.

以上两种重建EMCL线形的方法可以提高谱线拟 合的精度, 从而提高IMCL测量的精度, 但是它不能消 除主磁场不均匀性产生的谱线加宽和皮下脂肪对 


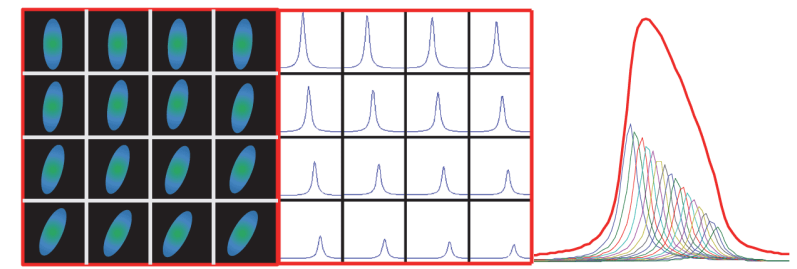

图 3 由DTI给出的纤维走向重建EMCL谱线线形的示意图 Figure 3 The reconstruction of EMCL lineshape based on fiber directions obtained from DTI

IMCL测量的影响.

\section{2 主磁场不均匀性}

理想假设下主磁场 $B_{0}$ 的空间分布是均匀的，谱线 的宽度很小 (约为 $0.02 \mathrm{ppm}$ ), 且小于EMCL与IMCL的 谱线间隔(约为 $0.2 \mathrm{ppm}$ ). 因此EMCL和IMCL可以被有 效分离. 但实际情况中, 主磁场 $B_{0}$ 的不均匀会导致谱线 加宽; 当谱线的半高宽达到 $0.2 \mathrm{ppm}$ 时, EMCL和IMCL 的谱线完全重合, 导致两者无法分辨.

主磁场不均匀性导致谱线加宽机制与上一节中所 描述的肌纤维分布造成的谱线加宽和畸变不同，它可 以被看作是谱线加宽函数对理想谱线的卷积. 针对该 问题, 本文作者之一提出了SPREAD(spectral resolution amelioration by deconvolution)方法 ${ }^{[34,35]}$, 可以部分地消 除磁场加宽的影响，改善分辨率．其原理是利用结构 MRI的相位信息，重建主磁场强度分布图(field map), 进而重建谱线的加宽函数; 用其对实测MRS谱线退卷 积，从而改善 EMCL与IMCL的可区分性。通过 SPREAD处理后, EMCL和IMCL的谱线线宽减小, 谱 线的可分辨性增强，且对所有的谱线都一致有效(图 $4 \mathrm{~A}$ 和 $\mathrm{B})$.

图4A采用大脑磁共振谱线作为研究对象, 采用 SPREAD方法改善谱线的可区分性：谱线为应用 SPREAD前, 由于谱线的分辨率差且存在线形畸变, $\mathrm{Cr}$ 和 $\mathrm{Ch}$ 的谱线不能准确地拟合和探测; 右侧谱线为应用 SPREAD方法后谱线的线宽变窄，分辨率和线形都得 到改善，使得谱线能准确地拟合(取自Dong和Peter$\operatorname{son}^{[36]}$ ). 图4B采用小腿肌质子波谱作为研究对象, 利 用SPREAD方法改善谱线加宽: 左上图是场图, 左下图 是原始的谱线, EMCL与IMCL严重重叠; 右下侧为经 SPREAD处理后的谱线, EMCL和IMCL的可分辨性 增强.
这种消除主磁场不均匀所致谱线加宽的方法并不 能消除因体磁化率效应因肌纤维方向导致的谱线畸变 和加宽 (见2.1节). 虽然主磁场不均匀谱线加宽与肌纤 维方向不均匀谱线加宽的机制不同，但是这两种加宽 的程度都与MRS体素的大小有关. 减小体素的体积可 以有效地减小谱线加宽 ${ }^{[26]}$. 但是, 对单体素MRS而言, 体素体积的减小伴随着信噪比的降低. 为获得足够的 信噪比, 采样时间往往需要增加. 更有效地提高谱线 分辨率的手段是采用多体素的高分辨波谱成像 ${ }^{[37,38]}$ 或 是超高场 $(>4 \mathrm{~T})$ 的 $M R S^{[39,40]}$. 以文献[40]为例, 单体素 MRS的体积只有 $3 \mathrm{~cm}^{3}$, 仍能获得较高分辨率和信噪比 的波谱. 而场强为 $4 \mathrm{~T}$ 的波谱成像的体素体积已经可以 小于 $1 \mathrm{~cm}^{3}$. 在如此小的空间内, 场的不均匀性和肌纤 维的分散性都大为减小, 使谱分辨率相应提高.

\section{3 皮下脂肪信号干扰}

皮下脂肪含量较高, 通常对应着较强的EMCL信 号(特别是潜在的糖尿病患者), 会对相邻肌肉的IMCL 信号造成污染, 甚至淹没IMCL信号 ${ }^{[33]}$, 如图5所示. 图5 中显示了小腿肌肉内三种不同位置的IMCL信号与 EMCL信号的关系. 左侧在包含细胞外脂质以及包含 筋膜的体元中, EMCL信号强度很高, 几乎淹没了 IMCL信号, 很难提取出来.

在单体素的MRS应用中，为了减少皮下脂肪信号 对IMCL测量的影响，可以选取离开皮下脂肪位置的 体素. 由于单体素MRS序列有很好的定域性, 这很容 易实现. 而磁共振波谱成像存在“点扩散函数”现象, 导致信号的定域性较差, 相邻体素之间有信号污染 ${ }^{[41]}$. 由于皮下脂肪的信号很强, 它对IMCL信号的污染可高 达2 3个体素.

早期抑制MRS中皮下脂肪信号影响的方法有如 下几种. 首先是一种实验方法, 称为外围空间饱和法 (outer volume suppression, OVS). 它是在轴向面MR图 像上, 沿着皮下脂肪布下若干条饱和激发带, 对脂肪信 号抑制. 这种方法可以有效地消除皮下脂肪信号的污 染. 其缺点是OVS饱和激发带也会覆盖一些肌肉区域. 一种简单的后处理方法是在对原始信号进行空间傅里 叶图像重建之前进行空间滤波. 文献[41]系统研究了 空间滤波函数的污染抑制效果. 这种方法的缺点是以 牺牲整个波谱图像的空间分辨率来抑制远程的信号污 染, 即皮下脂肪的污染距离减小了, 但整个图像的空间 


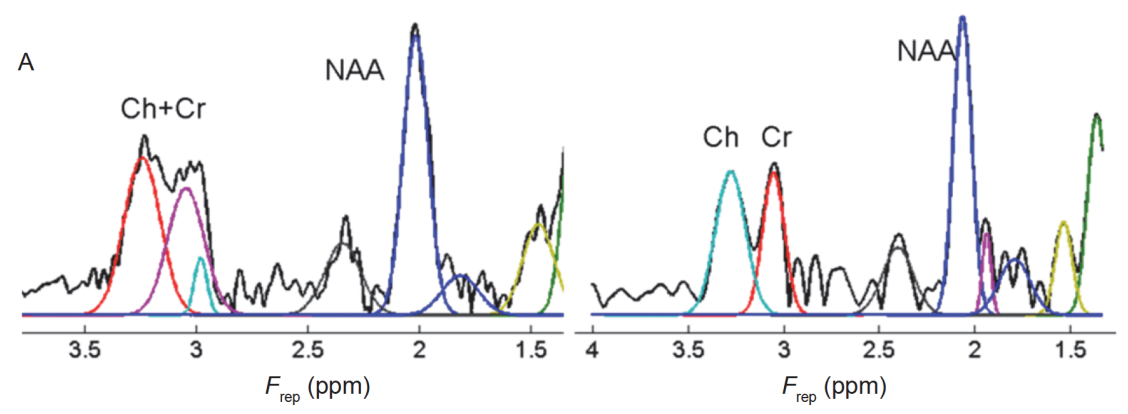

B

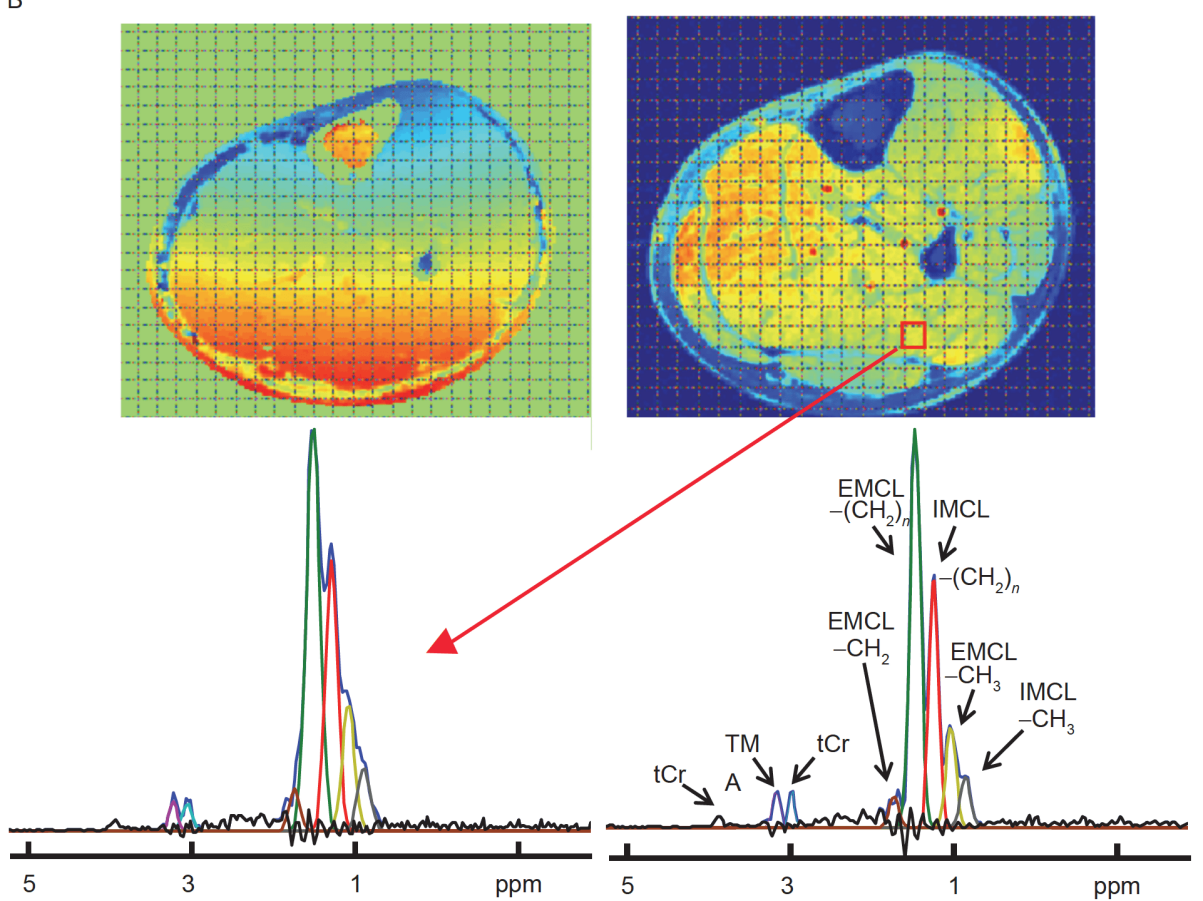

图 4 使用SPREAD方法前后磁共振谱可区分性对比效果图 ${ }^{[35]}$. A: 大脑质子磁共振谱 ${ }^{[36]}$; B: 小腿肌质子磁共振谱

Figure 4 Comparisons of the MR spectra before and after applying the SPREAD method ${ }^{[35]}$. A: ${ }^{1} \mathrm{H}$ MR spectra of the brain ${ }^{[36]}$; B: ${ }^{1} \mathrm{H}$ MR spectra of the calf muscle

分辨率却降低了. 另一种后处理方法是“脂肪外推法”. 点扩散函数现象是由于MRS的k-空间采样矩阵过小导 致的(如16×16或32×32). “脂肪外推法”以迭代算法不 断地将脂肪的 $\mathrm{k}-$ 空间外推，以减小点扩散函数的影响. 文献[41]研究了皮下脂肪信号对IMCL信号的污染并 研究了应用脂肪外推法的效果.

在前期的研究中，针对皮下脂肪、骨髓等高浓度 脂肪对相邻IMCL信号的污染问题，本课题组提出LESLIM方法 ${ }^{[30]}$ 消除皮下脂肪谱线的污染. 其方法是利 用脂肪MRI、结构MRI图像对皮下脂肪及骨髓进行分 割, 利用图像谱线定位(SLIM)方法重建皮下脂肪信号, 然后将它从原 ${ }^{1} \mathrm{H} \mathrm{MRS}$ 信号中剔除. 在不影响肌肉内脂
质信号谱线线形的情况下，抑制了皮下脂肪干扰信号 对附近肌群IMCL信号的干扰, 如图6所示. 图6中分别 选取小腿筋肉内 6 个不同的观测点，获得其对应的 ${ }^{1} \mathrm{H}$ MRS信号. 通过LE-SLIM方法, 对细胞外脂肪信号 EMCL进行抑制. 比较抑制前后，6个观测点的信号可 区分性，可以发现，该方法有效地改善了EMCL与 IMCL的分裂程度, 但它不能消除EMCL与IMCL的谱 线重叠、难以分离的问题 ${ }^{[42 ~ 44]}$.

综上所述，针对影响IMCL测量精度的各种因素， 学者们分别提出不同的解决思路, 但仍存在着一定的 局限性. 只有联合使用这些技术才能较全面地克服不 利因素的影响, 提高IMCL的测量精度. 


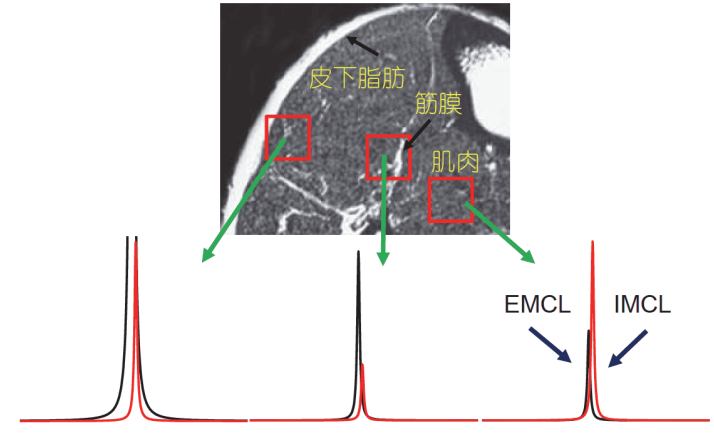

图 5 皮下脂肪信号对附近肌细胞内脂质IMCL信号造成污 染示意图

Figure 5 Contamination of the subcutaneous fat signal to the IMCL signals of the muscle
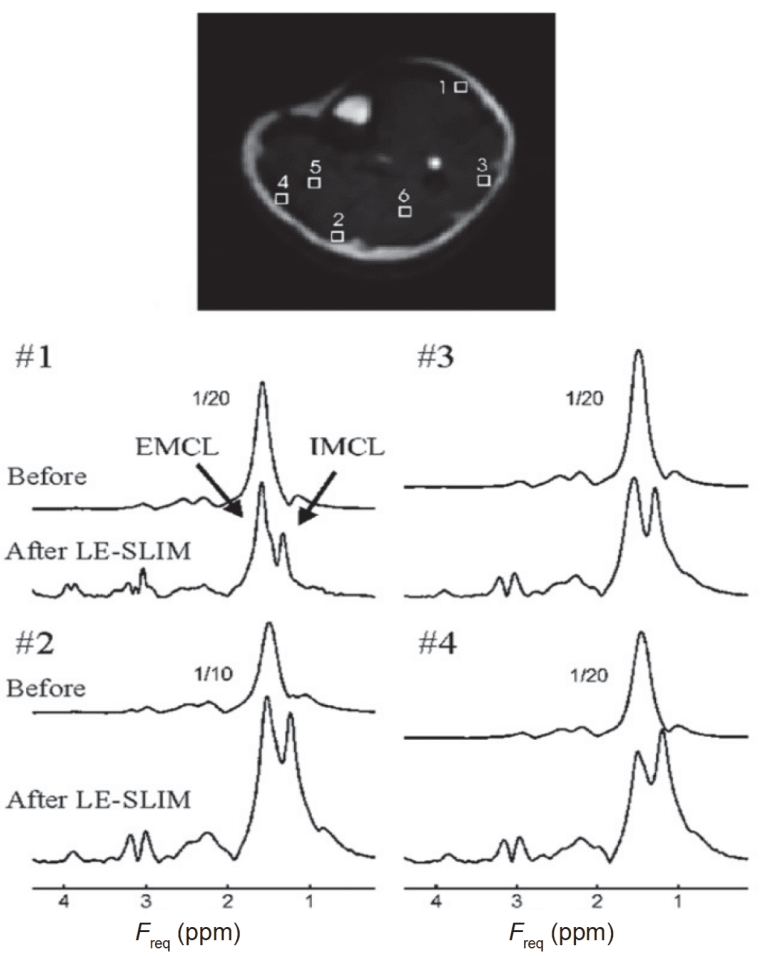

\#4
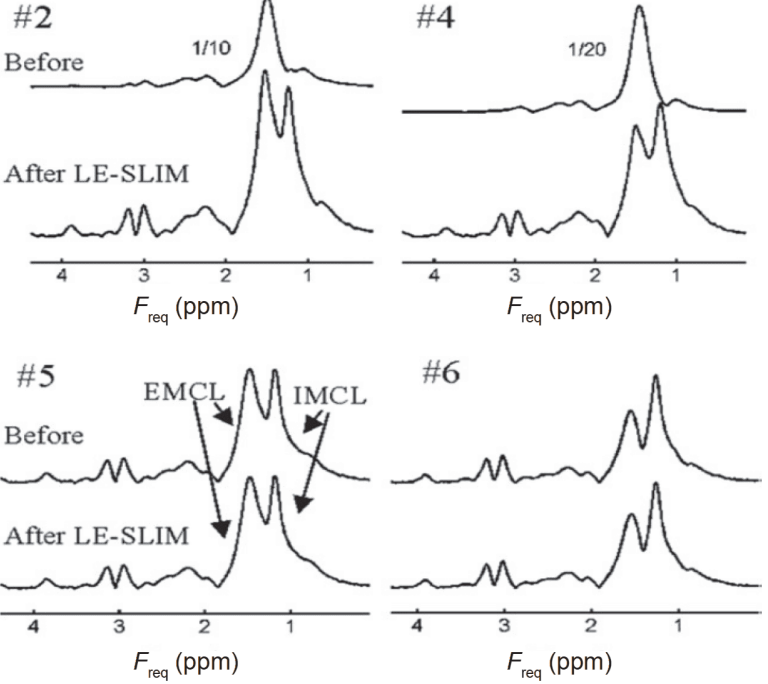

图 6 LE-SLIM方法消除皮下脂肪信号对IMCL干扰的效果 图 $^{[30]}$

Figure 6 The elimination of the contamination of subcutaneous fat signal to IMCL signal using the LE-SLIM method ${ }^{[30]}$

\section{IMCL测量技术面临的挑战及新的机遇}

\section{1 面临的挑战}

(1) 精确定量化的IMCL测量. 在IMCL精确定量化 测定过程中，不论是内部参照物信号还是外部参照物 信号，都必须与将它们的纵向弛豫时间 $T_{1}$ 和横向弛豫 时间 $T_{2}$ 有关的衰减矫正到绝对量化值上，才能准确地 估算出IMCL含量的具体数值. 而获得 $T_{1}$ 和 $T_{2}$ 的量化值 非常耗时 ${ }^{[29,45]}$. 为此, 缺乏 $T_{1}$ 和 $T_{2}$ 的准确数值使得 IMCL含量测定方法对于临床应用来说并不实用. 目 前使用的一种折中方法是采用已报道文献中测得的 $T_{1}$ 和 $T_{2}$ 值. 但这忽视了个体及不同病理生理状态下 $T_{1}$ 和 $T_{2}$ 值的差异性，以及由此引入的误差 ${ }^{[19,29,31,45,46]}$. 这不 符合个性化医学和精准医学的要求.

（2）可重复性和可靠性是评价任何测量技术及其 结果的重要指标. 由于磁共振波谱成像空间分辨率低 (体元的体积 $>1 \mathrm{~cm}^{3}$ ), 部分容积效应是IMCL波谱测不 准的一个关键因素. 关于小腿肌细胞内脂肪磁共振测 量技术的可重复性和可靠性的研究报道中，在 1.5 或 $3.0 \mathrm{~T}$ 的仪器上 IMCL 的测量精度的变化范围为 $20 \% \sim 50 \%{ }^{[37,42 \sim 44]}$, 而测量的可重复性的变化范围为 $10 \% \sim 20 \%{ }^{[44]}$. 这里涉及实验的参数如MRS空间分辨率 以及受试者的病理生理状况等问题.

（3）当肥胖糖尿病患者的肌纤维之间及肌纤维内 渗入的脂肪组织(intramuscular adipose tissue)的含量 很高时，其谱线会与IMCL有严重交叠，这会给IMCL 的精确测量带来不利的影响. 为此, 结合其他MR技术 获得肌纤维之间脂肪组织的分布情况, 将为IMCL测量 带来新的机遇 ${ }^{[47]}$.

\section{2 新的机遇}

(1) 磁共振指纹波谱技术. 近年来兴起的磁共振指 纹技术是一项可以同时获得多组织量化参数图(如 $T_{1}$, $T_{2}$, 质子密度等)的全新MR成像技术 ${ }^{[48,49]}$. 该项技术已 经在其他的MRI成像模态中得到推广，包括磁共振波 谱指纹技术.

图7是一个传统的磁共振波谱采样序列. 它通过三 个梯度场脉冲和射频脉冲实现空间定位，采集时域磁 共振波谱信号FID(free induced decay). 为提高信噪比, 采样需要重复累计若干次. FID信号经傅里叶变换即 成为所需的频域波谱信号. 在信号重复累加过程中, 


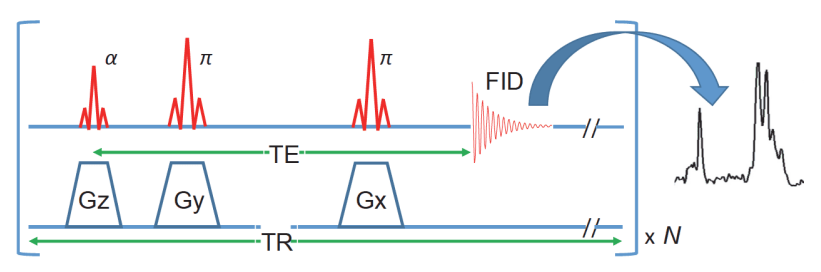

图 7 传统 ${ }^{1} \mathrm{H}$ MRS信号产生示意图

Figure 7 A diagram of traditional ${ }^{1} \mathrm{H}$ MRS sequence

序列的参数如重复时间(repetition time, TR)、回波时 间(time of echo, TE)、倾倒角(flip angle, FA) 都是固定 不变的; 所有的重复信号都有统一的 $T_{1}, T_{2}$ 加权因子.

磁共振波谱指纹的原理如图8所示. 它需要多次采 集FID信号, 且每次的TR, TE和FA参数均不同. 因此, 所有信号的 $T_{1}, T_{2}$ 加权因子都不相同, 使得每次采集的 EMCL, IMCL谱峰的强度不同. 另一方面, 我们又利用 布洛赫 $(\mathrm{BLOCH})$ 方程和给定的 TR, TE, FA序列参数 (图8), 计算EMCL, IMCL在各种可能的 $T_{1}, T_{2}$ 以及频率 参数下的信号. 每个信号称为与其参数相对应的一个 指纹。这些信号的集合称为“字典”。把实际测得的 EMCL和IMCL谱线序列与“字典”中的指纹比对，搜索 出相同者, 即可得到它所对应信号的参数, 如信号强 度, $T_{1}, T_{2}$ 等.

磁共振指纹波谱技术可以获得 $T_{1}, T_{2}$ 精确定量化 数值, 这对于IMCL的精确定量化测量至关重要. 与传 统MRS技术仅能提供一个峰面积与分子浓度成正比 的波谱相比，磁共振波谱指纹图谱可以在不需要额外 测量的情况下提供分子的 $T_{1}$ 和 $T_{2}$ 值. 这将大大方便 IMCL的精确定量化测量. 但目前尚未见磁共振波谱 指纹在IMCL测量中的应用. 还需要特别指出的是, $T_{1}$, $T_{2}$ 的精确量化技术不仅可用于IMCL的定量测量, 它们 本身还具有重要的临床研究价值 ${ }^{[50]}$.

(2) 高分辨率MRS成像技术. 目前多通道线圈 $(>32$ 个)已经在临床磁共振成像仪中广泛使用 ${ }^{[29]}$. 利用多线 圈并行成像的高灵敏度可以在不增加扫描时间的情况 下提高成像的空间分辨率. 同样, 高场 $(>3 \mathrm{~T})$ 磁共振成 像仪的高信噪比也可以使MRS成像技术向高分辨率 的方向发展. 最新的报道己经把MRS观测体元的大小 降到 $30 \mathrm{~mm}^{3}$. 这样, 很多不利于IMCL测量的因素都会 大为减少, 从而降低容积效应对信号测量的影响. 为 此，高分辨率的MRS技术，通过增加线圈个数及高场 磁共振成像技术，提高空间分辨能力，是降低容积效
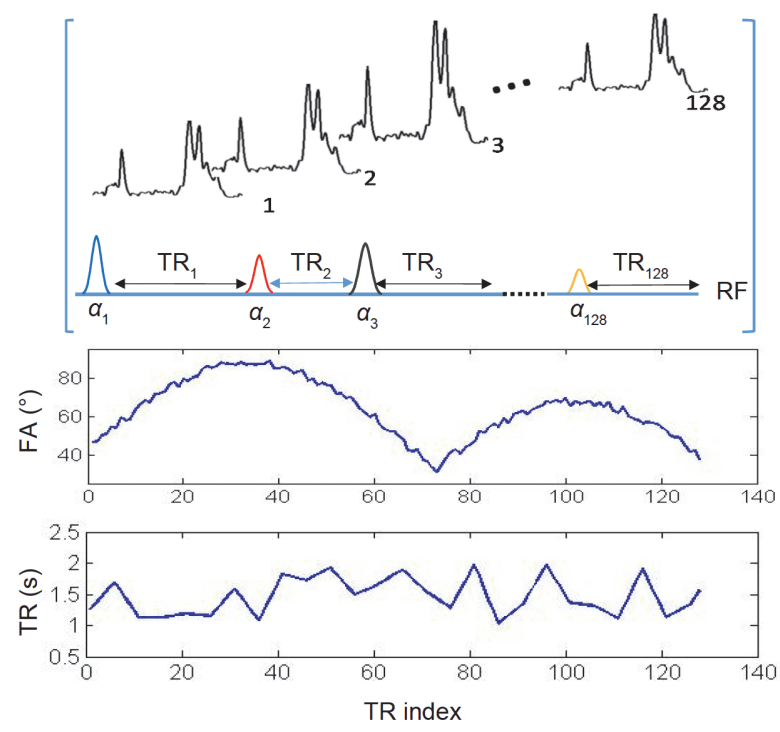

图 8 磁共振波谱指纹技术原理示意图

Figure 8 A diagram of magnetic resonance spectrum fingerprinting technology

应的关键.

(3) 多模态磁共振技术的发展. 准确重建EMCL谱 线线形, 需要结合小腿肌肉群中肌肉、脂肪、水分等 物质的分布情况, 分析体磁化率对EMCL谱线的加 宽、畸变效应. 为此, 综合运用多模态MRI(结构 MRI、脂肪MRI、弥散张量成像DTI以及核磁共振波 谱成像MRSI)技术，通过多样化的信息获取方式和多 维度、多尺度的测量途径, 使得获取小腿肌群结构、 功能及代谢物含量信息, 重构小腿肌群脂肪分布及肌 纤维走向, 分析体磁化率效应与氢谱线形畸变之间关 系成为可能, 是克服EMCL谱线加宽, 畸变效应对 IMCL造成干扰的关键.

\section{4 总结与展望}

本文围绕质子核磁共振波谱测量小腿肌内脂含量 的基本原理、技术上的发展、面临的挑战以及未来技 术发展的方向展开分析与讨论. 与传统方法相比, 质子 核磁共振波谱测量小腿肌内脂含量的方法具有不可比 拟的优势, 是未来个性化医学和精准医学的重要检测 手段. 经过 20 多年的发展, 这一技术已经日趋成熟. 但 在糖尿病基础研究和临床诊断上的应用还相对落后. 为此, 将该技术积极推广于糖尿病的发现、诊断和治 
疗及其相关领域还有待于进一步的研究.

另外, 美国糖尿病学会 ${ }^{[51 ~ 53]}$ 和美国心脏学会分 别发表了共识声明，肯定了结构性锻炼对 II 型糖尿 病预防护理和治疗的积极作用，并对健康人和 II 型 糖尿病患者的结构性锻炼提出了指导性的建议。根 据已知的细胞内脂质与糖尿病的关联，本文推测结 构化锻炼前后人的细胞内脂质应发生与糖尿病状况
相应的变化, 但目前尚未有相关研究报道 ${ }^{[5456]}$. 为此, 将质子核磁共振波谱的优势和本文介绍的相关新技 术，用于个体或群体的结构化锻炼优化研究，测量和 比对结构化锻炼前后人的细胞内脂质含量与糖尿病 状况改变之间的关系, 将对 II 型糖尿病的个性化精准 管理、控制和改善具有重要的推动作用, 是未来研究 的重要方向.

\section{参考文献}

1 Bucci M, Huovinen V, Guzzardi M A, et al. Resistance training improves skeletal muscle insulin sensitivity in elderly offspring of overweight and obese mothers. Diabetologia, 2016, 59: 77-86

2 Carlier P G, Marty B, Scheidegger O, et al. Skeletal muscle quantitative nuclear magnetic resonance imaging and spectroscopy as an outcome measure for clinical trials. J Neuromuscul Diseases, 2016, 3: 1-28

3 Vaisy M, Szlufcik K, De Bock K, et al. Exercise-induced, but not creatine-induced, decrease in intramyocellular lipid content improves insulin sensitivity in rats. J Nutrit Biochem, 2011, 22: 1178-1185

4 Roglic G. WHO Global report on diabetes: A summary. Int J Non-Commun Dis, 2016, 1: 3

5 Li X, Peng Y N, Zhou Z G. Latent autoimmune diabetes in adults in China: Clinical features and epidemiology. Sci Sin-Vitae, 2018, 48: 847-854

6 Paschou S A, Leslie R D. Personalizing guidelines for diabetes management: Twilight or dawn of the expert? BMC Med, 2013, 11: 161

7 Thomas P P M, Alshehri S M, van Kranen H J, et al. The impact of personalized medicine of type 2 diabetes mellitus in the global health context. Personalized Med, 2016, 13: 381-393

8 Shulman G I. Cellular mechanisms of insulin resistance. J Clin Invest, 2000, 106: 171-176

9 Warram J H, Martin B C, Krolewski A S, et al. Slow glucose removal rate and hyperinsulinemia precede the development of type II diabetes in the offspring of diabetic parents. Ann Intern Med, 1990, 113: 909-915

10 O'Rahilly S, Savill J. Science, medicine, and the future non-insulin dependent diabetes mellitus: The gathering storm. BMJ, 1997, $314: 955$

11 Jacob S, Machann J, Rett K, et al. Association of increased intramyocellular lipid content with insulin resistance in lean nondiabetic offspring of type 2 diabetic subjects. Diabetes, 1999, 48: 1113-1119

12 Virkamäki A, Korsheninnikova E, Seppälä-Lindroos A, et al. Intramyocellular lipid is associated with resistance to in vivo insulin actions on glucose uptake, antilipolysis, and early insulin signaling pathways in human skeletal muscle. Diabetes, 2001, 50: 2337-2343

13 Krssak M, Roden M. The role of lipid accumulation in liver and muscle for insulin resistance and type 2 diabetes mellitus in humans. Rev Endocr Metab Disord, 2004, 5: 127-134

14 Machann J, Häring H, Schick F, et al. Intramyocellular lipids and insulin resistance. Diabetes Obes Metab, 2004, 6: 239-248

15 Beha A, Juretschke H P, Kuhlmann J, et al. Muscle type-specific fatty acid metabolism in insulin resistance: An integrated in vivo study in Zucker diabetic fatty rats. Am J Physiol-Endocrinol Metab, 2006, 290: E989-E997

16 White L J, Ferguson M A, McCoy S C, et al. Cardiovascular/non-insulin-dependent diabetes mellitus risk factors and intramyocellular lipid in healthy subjects: A sex comparison. Metabolism, 2006, 55: 128-134

17 http://www.uclh.nhs.uk/PandV/PIL/Patient informationleaflets/Muscle Biopsy.pdf. 2015

18 http://www.hopkinsmedicine.org/healthlibrary/test_procedures/orthopaedic/muscle_biopsy_92,P07671. 2013

19 Alger J R. Quantitative proton magnetic resonance spectroscopy and spectroscopic imaging of the brain. Top Magn Reson Imag, 2010, 21: 115128

20 Hwang J H, Stein D T, Barzilai N, et al. Increased intrahepatic triglyceride is associated with peripheral insulin resistance: In vivo MR imaging and spectroscopy studies. Am J Physiol-Endocrinol Metab, 2007, 293: E1663-E1669

21 Khuu A, Ren J, Dimitrov I, et al. Orientation of lipid strands in the extracellular compartment of muscle: Effect on quantitation of intramyocellular lipids. Magn Reson Med, 2009, 61: 16-21

22 Valaparla S K, Gao F, Daniele G, et al. Fiber orientation measurements by diffusion tensor imaging improve hydrogen-1 magnetic resonance 
spectroscopy of intramyocellular lipids in human leg muscles. J Med Imag, 2015, 2: 026002

23 Reeder S B, Pineda A R, Wen Z, et al. Iterative decomposition of water and fat with echo asymmetry and least-squares estimation (IDEAL): Application with fast spin-echo imaging. Magn Reson Med, 2005, 54: 636-644

24 Hwang J H, Choi C S. Use of in vivo magnetic resonance spectroscopy for studying metabolic diseases. Exp Mol Med, 2015, 47: e139

25 Trout K K, Homko C, Tkacs N C. Methods of measuring insulin sensitivity. Biol Res Nursing, 2007, 8: 305-318

Boesch C, Slotboom J, Hoppeler H, et al. In vivo determination of intra-myocellular lipids in human muscle by means of localized ${ }^{1} \mathrm{H}-\mathrm{MR}$ spectroscopy. Magn Reson Med, 1997, 37: 484-493

27 Vermathen P, Saillen P, Boss A, et al. Skeletal muscle ${ }^{1} \mathrm{H}$ MRSI before and after prolonged exercise. I. Muscle specific depletion of intramyocellular lipids. Magn Reson Med, 2012, 68: 1357-1367

28 Ortiz-Nieto F, Johansson L, Ahlström H, et al. Quantification of lipids in human lower limbs using yellow bone marrow as the internal reference: Gender-related effects. Magn Reson Imag, 2010, 28: 676-682

29 Weis J, Johansson L, Ortiz-Nieto F, et al. Assessment of lipids in skeletal muscle by high-resolution spectroscopic imaging using fat as the internal standard: Comparison with water referenced spectroscopy. Magn Reson Med, 2008, 59: 1259-1265

30 Dong Z, Hwang J H. Lipid signal extraction by SLIM: Application to ${ }^{1} \mathrm{H}$ MR spectroscopic imaging of human calf muscles. Magn Reson Med, 2006, 55: 1447-1453

31 Dong Z. Proton MRS and MRSI of the brain without water suppression. Prog Nucl Magn Reson Spectr, 2015, 86-87: 65-79

32 Karampinos D C, Yu H, Shimakawa A, et al. Chemical shift-based water/fat separation in the presence of susceptibility-induced fat resonance shift. Magn Reson Med, 2012, 68: 1495-1505

33 Boesch C, Machann J, Vermathen P, et al. Role of proton MR for the study of muscle lipid metabolism. NMR Biomed, 2006, 19: 968-988

34 Dong Z, Zhang Y, Liu F, et al. Improving the spectral resolution and spectral fitting of ${ }^{1} \mathrm{H}$ MRSI data from human calf muscle by the SPREAD technique. NMR Biomed, 2014, 27: 1325-1332

35 Dong Z, Liu F, Duan Y, et al. Enhancing spectral resolution in ${ }^{1} \mathrm{H}$ MRSI of human calf muscles by SPREAD. In: Proceedings of the 18th Annual Meeting of ISMRM. Stockholm, 2010

36 Dong Z, Peterson B S. Spectral resolution amelioration by deconvolution (SPREAD) in MR spectroscopic imaging. J Magn Reson Imag, 2009, 29: $1395-1405$

37 Hwang J H, Pan J W, Heydari S, et al. Regional differences in intramyocellular lipids in humans observed by in vivo ${ }^{1} \mathrm{H}-\mathrm{MR}$ spectroscopic imaging. J Appl Physiol, 2001, 90: 1267-1274

38 Weis J, Bruvold M, Ortiz-Nieto F, et al. High-resolution echo-planar spectroscopic imaging of the human calf. PLoS ONE, 2014, 9: e87533

39 Ren J, Sherry A D, Malloy C R. ${ }^{1}$ H MRS of intramyocellular lipids in soleus muscle at 7 T: Spectral simplification by using long echo times without water suppression. Magn Reson Med, 2010, 64: 662-671

40 Golla S, Ren J, Malloy C R, et al. Intramyocellular lipid excess in the mitochondrial disorder MELAS. Neurol Genet, 2017, 3: e160

41 Cui M H, Hwang J H, Tomuta V, et al. Cross contamination of intramyocellular lipid signals through loss of bulk magnetic susceptibility effect differences in human muscle using ${ }^{1}$ H-MRSI at 4 T. J Appl Physiol, 2007, 103: 1290-1298

42 Weis J, Johansson L, Ortiz-Nieto F, et al. Assessment of lipids in skeletal muscle by LCModel and AMARES. J Magn Reson Imag, 2009, 30: $1124-1129$

43 Shen W, Mao X, Wolper C, et al. Reproducibility of single- and multi-voxel ${ }^{1} \mathrm{H}$ MRS measurements of intramyocellular lipid in overweight and lean subjects under conditions of controlled dietary calorie and fat intake. NMR Biomed, 2008, 21: 498-506

44 Torriani M, Thomas B J, Halpern E F, et al. Intramyocellular lipid quantification: Repeatability with ${ }^{1} \mathrm{H}$ MR spectroscopy. Radiology, 2005, 236: $609-614$

45 Wang L, Salibi N, Wu Y, et al. Relaxation times of skeletal muscle metabolites at 7 T. J Magn Reson Imag, 2009, 29: 1457-1464

46 Sharma P, Martin D R, Pineda N, et al. Quantitative analysis of T2-correction in single-voxel magnetic resonance spectroscopy of hepatic lipid fraction. J Magn Reson Imag, 2009, 29: 629-635

47 Ogawa M, Lester R, Akima H, et al. Quantification of intermuscular and intramuscular adipose tissue using magnetic resonance imaging after neurodegenerative disorders. Neural Regen Res, 2017, 12: 2100

48 Kulpanovich A, Tal A. The application of magnetic resonance fingerprinting to single voxel proton spectroscopy. NMR Biomed, 2018, 31: e4001

49 Li M, Dong Z C, Zhang X W, et al. The technique of magnetic resonance fingerprinting and its latest development (in Chinese). Sci Sin Vitae, 
2019, 49: 28-40 [李敏, 董正超, 张学武 等. 磁共振指纹成像技术及其最新进展. 中国科学: 生命科学, 2019, 49: 28-40]

50 Azzabou N, Loureiro de Sousa P, Caldas E, et al. Validation of a generic approach to muscle water $T_{2}$ determination at $3 \mathrm{~T}$ in fat-infiltrated skeletal muscle. J Magn Reson Imag, 2015, 41: 645-653

51 Colberg S R, Sigal R J, Yardley J E, et al. Physical activity/exercise and diabetes: A position statement of the American Diabetes Association. Dia Care, 2016, 39: 2065-2079

52 Fox C S, Golden S H, Anderson C, et al. Update on prevention of cardiovascular disease in adults with type 2 diabetes mellitus in light of recent evidence. Circulation, 2015, 132: 691-718

53 Fox C S, Golden S H, Anderson C, et al. Update on prevention of cardiovascular disease in adults with type 2 diabetes mellitus in light of recent evidence: A scientific statement from the American Heart Association and the American Diabetes Association. Dia Care, 2015, 38: 1777-1803

54 Zehnder M, Ith M, Kreis R, et al. Gender-specific usage of intramyocellular lipids and glycogen during exercise. Med Sci Sports Exercise, 2005, 37: $1517-1524$

55 Rouffet D, Villars C, Fissoune R, et al. Intramyocellular lipid variations in active older men: Relationship with aerobic fitness. Acta Physiol, 2013, 207: 516-523

56 Bajpeyi S, Reed M A, Molskness S, et al. Effect of short-term exercise training on intramyocellular lipid content. Appl Physiol Nutr Metab, 2012, 37: $822-828$

\title{
The principle, present and prospect of IMCL measurement based on ${ }^{1}$ H MRSI technology
}

\author{
LI Min ${ }^{1,2}$, ZHANG XueWu ${ }^{1,2}$, SHEN WenRong ${ }^{3}$, DENG ZhiXiang ${ }^{1}$ \& DONG ZhengChao ${ }^{2,4}$ \\ 1 College of Internet of Things Engineering, Hohai University, Changzhou 213022, China; \\ 2 Division of Molecular Imaging, Department of Psychiatry, Columbia University, New York 10032, USA; \\ 3 Department of Radiology, Jiangsu Provincial Cancer Hospital, Nanjing 210009, China; \\ 4 New York State Psychiatric Institute, New York 10032, USA
}

It has been found that, as a main mechanism for diabetes, insulin resistance may have appeared more than a decade before the onset of diabetes. Therefore, early detection of insulin resistance has vital significance for the early prevention, diagnosis, and treatment of diabetes. The content of intramyocellular lipid (IMCL) in human calf muscle has close and complex relationship with insulin resistance and metabolic disorders and, therefore, is associated with type 2 diabetes mellitus and even a variety of mental diseases. However, the current "gold standard" for IMCL measurement is the invasive biopsy technology, which is not suitable for large-scale basic research and long-term clinical applications. IMCL measurement technology based on proton magnetic resonance spectroscopy and spectroscopic imaging $\left({ }^{1} \mathrm{H}\right.$ MRSI) provides a radiation-free, non-invasive, and high-precision detection method. More than this, ${ }^{1} \mathrm{H}$ MRSI has been expected to clarify the relationship between IMCL content and insulin resistance, as well as the relationship between insulin resistance and type 2 diabetes mellitus, and has the potential to be applied in clinical practice. This paper describes and summarizes the technical development of ${ }^{1} \mathrm{H}$ MRSI-based IMCL content measurement. It also points out the challenges and the latest solutions of this technique from the aspects of physiological and structural characteristics of calves, muscle fiber direction, the bulk fat distribution, and the demand for precise quantification of IMCL content. In addition to reviewing the latest technical solutions to these problems, we particularly discuss the combination of ${ }^{1} \mathrm{H}$ MRSI with some new technologies such as multi-mode magnetic resonance imaging (MRI) and magnetic resonance fingerprinting spectroscopy (MRFS), which will synergically provide more diversified, multi-dimensional and multi-scale measurement method for high-precision measurement of IMCL.

magnetic resonance spectroscopy and spectroscopic imaging, intramyocellular lipid, extra-myocellular lipids, diabetes, myofiber direction, MRI, ${ }^{1} \mathrm{H}$ MRS

doi: $10.1360 / \mathrm{N} 052018-00278$ 